\title{
Late-onset hypogonadism or ADAM: treatment
}

\author{
Hipogonadismo masculino taRdio ou DAEM: tRATAmEnto \\ Authorship: Brazilian Society of Endocrinology; Brazilian Society of Urology \\ Participants: Martits AM, Costa EMF, Nardi AC, Nardozza Jr. A, Facio Jr. FN, Faria G, Bernardo WM \\ Conflict of interest: no conflict of interest informed.
}

Final draft: March 21 ${ }^{\text {st }}, 2014$

http://dx.doi.org/10.1590/1806-9282.60.05.005

The Guidelines Project, an initiative of the Brazilian Medical Association, aims to combine information from the medical field in order to standardize procedures to assist the reasoning and decision-making of doctors.

The information provided through this project must be assessed and criticized by the physician responsible for the conduct that will be adopted, depending on the conditions and the clinical status of each patient.

\section{Description Of the eVIDENCE COLLECtion METHOD}

The recommendations were supported by the evidence obtained in the Medline, Embase, Lilacs and Cochrane databases, using the following strategies: ((((()aged OR aging) AND (androgens/deficiency OR hypogonadism OR testosterone/deficiency)) AND (hormone replacement therapy) AND (aging/blood OR androgens/blood* OR androgens OR androstenedione/blood OR body mass index OR body Weight OR dehydroepiandrosterone/blood* OR dehydroepiandrosterone sulfate/blood* OR dihydrotestosterone/ blood OR gonadal steroid hormones/blood* OR gonadotropins/blood OR gonadotropins OR pituitary hormones OR hormones/blood* OR hormones/physiology OR hypogonadism/blood OR hypotalamo-hypophyseal system OR immunoassay OR luteinizing hormone OR luteinizing hormone/blood OR radioimmunoassay OR radioimmunoassay/methods OR radioimmunoassay/standards* OR reagent kits, diagnostic/standards OR sex hormone-binding globulin/analysis OR testosterone OR testosterone/ blood* OR testosterone/metabolism OR hematocrit OR liver function tests OR prostatic neoplasms OR continuity of patient care OR consultation OR monitoring))) AND (diagnosis/broad[filter]) AND (“last 5 years"[PDat]))) OR (((((aged OR aging) AND (androgens/deficiency OR hypogonadism OR testosterone/deficiency)) AND (hormone replacement therapy) AND (aging/blood OR androgens/ blood* OR androgens OR androstenedione/blood OR body mass indexOR body weight OR dehydroepiandrosterone/blood* OR dehydroepiandrosterone sulfate/blood* OR dihydrotestosterone/blood OR gonadal steroid hormones/blood* OR gonadotropins/blood OR gonadotro- pins OR pituitary hormones OR hormones/blood* OR hormones/physiology OR hypogonadism/blood OR hypotalamo-hypophyseal system OR immunoassay OR luteinizing hormone OR luteinizing hormone/blood OR radioimmunoassay OR radioimmunoassay/methods OR radioimmunoassay/standards* OR reagent kits, diagnostic/standards OR sex hormone-binding globulin/analysis OR testosterone OR testosterone/blood* OR testosterone/metabolism OR hematocrit OR liver function tests OR prostatic neoplasms OR continuity of patient care OR consultation OR monitoring))) AND (etiology/broad[filter]) (age OR aged OR aging) AND (andropause OR gonadal OR deficiency OR hypogonadism OR hypogonadal) AND (testosterone OR androsterone OR DHEA OR dehydroepiandrosterone $\mathrm{OR}$ androgen $\mathrm{OR}$ androgenic $\mathrm{OR}$ nandrolone OR replacement OR steroids OR anabolic OR sex OR hormone) AND (muscles OR strength OR cognitive OR function OR cognition OR mental OR performance OR diabetic OR diabetes OR obesity OR body OR fat OR obese OR mass OR hyperinsulinaemia OR adiposity OR libido OR sexual OR behavior OR quality of life OR life style OR lipids OR carbohydrates OR metabolism) (((age OR aged OR aging) AND (andropause OR gonadal OR deficiency OR hypogonadism OR hypogonadal) AND (testosterone OR androsterone OR DHEA OR dehydroepiandrosterone $\mathrm{OR}$ androgen $\mathrm{OR}$ androgenic $\mathrm{OR}$ nandrolone $\mathrm{OR}$ replacement OR steroids OR anabolic OR sex OR hormone))) AND (polycythemia OR prostatic neoplasms OR cardiovascular diseases OR liver diseases OR sleep apnea syndromes OR metabolic syndrome). 


\section{Grade OF RECOMmEndation AND STRENGTH OF EVIDENCE}

A: Experimental and observational studies with high consistency.

B: Experimental and observational studies with low consistency.

C: Case reports (non-controlled studies).

D: Opinions without critical evaluation, based on consensus, physiological studies, or animal models.

\section{Овjective}

To recommend evidence-based approaches to treat late-onset hypogonadism.

\section{INTRODUCTION}

The benefits of androgen replacement therapy in hypogonadal young men are well documented, especially given that the restoration of testosterone concentrations to normal limits maintains and restores sexual function, energy, mood, the development of muscle mass and increases bone mass. However, the cost/benefit relationship of testosterone replacement in Androgen Deficiency in the Aging Male (ADAM) remains controversial (A). ${ }^{1}$

\section{WHAT IS THE ROLE OF ANDROGEN REPLACEMENT THERAPY (ART) TO RESTORE BONE MASS, MUSCULAR STRENGTH AND BODY COMPOSITION?}

There is no doubt that ART has a positive effect on bone mass. The treatment with testosterone determines a significant improvement in bone mass in hypogonadal men of all ages $(\mathbf{B}){ }^{2}$ This effect becomes even more evident the longer the treatment time and the more severe the hypogonadism $(\mathbf{A}) .^{3}$

The effects of ART on muscular strength in men with ADAM are generally evaluated indirectly through analysis of the performance of physical activities and body composition. Therefore, the use of transdermal testosterone at a dose of $50 \mathrm{mg} /$ day for a 6 month period has been shown to be capable of increasing muscular strength and lean mass in patients, improving their physical function and quality of life (A). ${ }^{4}$ Direct evaluation of muscle size in elderly patients with chronic diseases with a loss in muscle mass and ADAM have shown that testosterone therapy in older patients leads to an increase in muscle size and improved muscular strength, improving performance in physical activities $(\mathbf{B}) .^{5}$

The most reproducible effect of testosterone treatment is the increase in lean mass both in hypogonadal patients and healthy males. Even if for a short period of time, transdermal testosterone treatment increases muscle mass in hypogonadal men aged over 40 years $(\mathbf{A})^{3}(\mathbf{B}){ }^{6}$

On the other hand, studies evaluating the reduction in body fat as a direct effect of testosterone treatment are controversial. A slight reduction in body fat in patients being treated has sometimes been observed (A). ${ }^{3}$ Testosterone treatment results in a significant reduction in waist circumference, which is an indicator of visceral fat (A). ${ }^{7}$ However, this benefit has not been shown in short acting treatments $(\mathbf{A}){ }^{8}$

The reduction in BMI and waist circumference may not occur in all patients, but the reduction in visceral fat is evident when analyzed directly using magnetic resonance imaging. Therefore, testosterone therapy selectively reduces the accumulation of visceral fat, though the change in total body composition is not observed in all patients $(\mathbf{B})^{6}(\mathbf{A}){ }^{9}{ }^{9}$

This discrepancy in the findings in relation to the effects of testosterone on the reduction of lean mass may be related to the dose or route of testosterone administration, or the direct effect of testosterone on the increase in lean mass. Ultimately, testosterone replacement improves the body composition of patients with low levels of the hormone $(\mathbf{B}){ }^{2}$

The use of GH in association with testosterone appears to present a synergic effect on the improvement in body composition, muscular strength and, consequently, the quality of life of men with ADAM, and can be a treatment option to be considered in such patients $(\mathbf{A}) \cdot{ }^{10,11}$

\section{Recommendation}

Testosterone replacement therapy improves bone mass, muscular strength and body composition in patients with ADAM and the effects increase with the length of treatment and severity of hypogonadism. The use of testosterone in patients with ADAM is strongly recommended to increase bone mass, muscular strength and body composition.

\section{WHAT IS THE ROLE OF ANDROGEN REPLACEMENT THERAPY (ART) TO RESTORE LIBIDO AND SEXUAL FUNCTION?}

The effectiveness of testosterone replacement therapy on sexual symptoms has been well established, regardless of the route of administration; nevertheless, the works are controversial in relation to the effectiveness of the oral route. Treatment with testosterone, regardless of the route of administration, improves and restores the libido in men with lower testosterone levels $(\mathbf{B})^{12-15}$ in the first three months of treatment $(\mathbf{C}) \cdot{ }^{16}$ However, testosterone does 
not seem to have an effect on the sexual function of eugonadal males $(\mathbf{B}) .{ }^{12}$ Long acting injectable testosterone has demonstrated greater tolerability and more physiological action (B) ${ }^{15}$ while the oral form was not effective in improving sexual symptoms (A). ${ }^{17}$

Studies suggest a direct relationship between free testosterone and vasodilation of the corpora cavernosa. As such, testosterone therapy presents a synergic effect on patients with ADAM that have responded partially to phosphodiesterase inhibitors, significantly improving erectile dysfunction in such patients $(\mathbf{B}) .^{12,13,18}$ Ultimately, when erectile dysfunction does not respond to testosterone treatment, the combination of phosphodiesterase inhibitors should be considered (B). ${ }^{2}$

Not only testosterone but also DHEA has been considered an important androgen for adequate sexual function, although its effectiveness in improving sexual symptoms and erectile dysfunction has not been demonstrated (A). ${ }^{19}$

\section{Recommendation}

Testosterone replacement therapy is recommended for improving libido and sexual function only in patients with low testosterone levels. The use of testosterone is recommended for improving the libido and sexual function of patients with ADAM.

\section{WhAT IS THE ROLE OF ANDROGEN REPLACEMENT THERAPY (ART) TO IMPROVE MOOD, QUALITY OF LIFE AND COGNITIVE FUNCTIONS?}

The influence of testosterone replacement therapy on the quality of life of older men has been widely evaluated. Although the majority of studies do not have good evidence, an important improvement on quality of life after testosterone replacement has been suggested in both hypogonadal and eugonadal men.

Comparing the quality of life of men with ADAM that use testosterone with those that do not, the improvement in the quality of life of the group using testosterone could be relative, i.e. determined by the decline in quality of life of the placebo group, suggesting a possible positive effect of testosterone on preventing the decline in quality of life with age (A). ${ }^{3}$ Furthermore, the improvement of physical function and control and somatic and sexual symptoms with testosterone replacement improves the quality of life of patients with ADAM, and may constitute an important treatment strategy in old age $(\mathbf{A})^{4}(\mathbf{B}){ }^{5}$

The effects of testosterone replacement on cognitive functions, mood and sense of wellbeing in men with ADAM are not yet clear. However, studies with excellent evidence have demonstrated that regardless of the route of administration, dose or treatment time, testosterone replacement does not affect cognitive function, mood or quality of life in men with $\operatorname{ADAM}(\mathbf{A})^{20,21}(\mathbf{B}) .^{22}$

\section{Recommendation}

Testosterone replacement improves mood and quality of life in men with ADAM; however, there is no evidence of a direct effect of testosterone over the above, or over cognitive function. It is strongly recommended not to use testosterone to specifically improve mood, quality of life or cognitive functions in men with ADAM.

\section{What IS THE INFLUENCE OF ANDROGEN REPLACEMENT THERAPY (ART) ON THE METABOLISM OF CARBOHYDRATES AND LIPIDS?}

Hyperinsulinemia and insulin resistance (IR) are antecedents of type 2 diabetes mellitus (T2DM) and metabolic syndrome, which in turn is characterized by IR associated with changes in lipid profile, among others. Type 2 diabetes is often associated with male hypogonadism, and it has also been suggested that testosterone replacement improves glycemic control as well as the body fat in patients with T2DM (B). ${ }^{23}$

Replacement therapy with injectable testosterone has been shown to be effective in improving insulin resistance and glycemic control with a significant reduction in fasting blood glucose and glycated hemoglobin (HbA1c) in hypogonadal men with type 2 diabetes $(\mathbf{A})^{7}$ or metabolic syndrome $(\mathbf{C}) .{ }^{16}$ Similar efficacy has also been observed in the metabolic syndrome parameters, with a significant reduction in waist circumference, blood pressure, total cholesterol, LDL and triglycerides and an increase in HDL levels, albeit without changes in the dietary patterns $(\mathbf{C}) .{ }^{16}$ An additional effect to that described above has been observed in the administration of long-acting testosterone. Patients with metabolic syndrome and ADAM achieved a dramatic reduction in the levels of insulin, leptin, HOMA-R and inflammatory markers such as IL-1 $\beta$, TNF $\alpha$ and PCR (A). ${ }^{9}$

The association of testosterone with an aromatase inhibitor leads to a significant increase in the testosterone/estradiol ratio and thereby a significant reduction in triglyceride levels in such patients. Therefore, this combination may be particularly useful in patients with hypertriglyceridemia. However, the safety of the use of this association has not yet been established (A). ${ }^{24}$

Ultimately, the effects of testosterone on carbohydrate and lipid metabolism are still uncertain. Testosterone treatment in hypogonadal men with type 2 diabetes and/or 
metabolic syndrome can lead to (yet unproven) benefits for their metabolic state $(\mathbf{B}){ }^{2}$

There is speculation about the role of the age-related decrease in DHEA on the metabolism of carbohydrates. The few studies that have addressed this issue suggest that DHEA has no effect on the metabolism of carbohydrates $(\mathbf{B}) .{ }^{25}$

\section{Recommendation}

The effects and benefits of testosterone replacement on the metabolism of carbohydrates and lipids are still controversial. Testosterone replacement is recommended in men with ADAM and metabolic syndrome as an adjunctive to improve the metabolic profile of such patients.

\section{What IS THE RISK OF ART IN RELATION TO EXACERBATING PROSTATE DISEASE?}

Unfortunately, to date there is no consensus on the response pattern of PSA to testosterone therapy. It has been argued that a significant increase in PSA after beginning testosterone replacement can be a sign of prostate cancer. However, most studies do not support this argument.

Historically, TRT has been absolutely contraindicated in men with a suspected or confirmed diagnosis of prostate cancer. There is evidence that TRT can stimulate growth and aggravate the symptoms of prostate cancer in men with active disease.

TRT causes a mild increase in PSA in most patients without prostatic changes and such increase does not depend on patient age, mode of replacement, or baseline PSA levels and total free testosterone $(\mathbf{B}) .{ }^{26}$ In intra-prostatic cancer patients with normalization of PSA after permanent brachytherapy or external beam radiation therapy who developed hypogonadism, testosterone treatment determined a slight increase in PSA with no signs of recurrence or progression of the cancer $(\mathbf{C}),{ }^{27,28}$ while in those submitted to radical prostatectomy, PSA levels did not change (B). ${ }^{29}$ In all these studies, testosterone levels were maintained within the normal range. On the other hand, when serum testosterone levels are kept above the normal values in patients previously treated for prostate cancer, even with the association of 5 - $\alpha$-reductase inhibitors, PSA levels rise significantly $(\mathbf{B}){ }^{30}$

In patients who received curative treatment for low risk localized prostate cancer with the radical prostatectomy and/or radiotherapy, with no evidence of active disease, the risks and benefits of TRT should be assessed. There is no consensus on the moment for determining consolidated healing treatments with this intention in localized prostate can- cer. Short-term studies with few patients suggest that after 1 year of treatment TRT can commence if the patient is cured.

The data available in the literature indicate that:

1. The incidence of prostate cancer in men with late-onset hypogonadism on TRT is no greater than the population in general $(\mathbf{B}) ;{ }^{31}$

2. TRT in older men with late-onset hypogonadism seems to have a slight effect on prostate tissue $(\mathbf{A}) ;{ }^{32}$

3. In patients with prostate cancer treated using radical prostatectomy, brachytherapy or radiotherapy, TRT can be used with caution, and patients should be constantly monitored $(\mathbf{C}) ; 2,28$

4. High doses of testosterone, even if associated with 5-alpha-reductase inhibitors, cause a significant increase in PSA $(\mathbf{B}) .^{30}$

The greatest risk of prostate cancer has been observed in men with higher estrone levels (A) ${ }^{33}$ however, studies with a higher number of patients should be conducted to confirm this observation.

\section{Recommendation}

Maintaining testosterone at physiological levels increases PSA levels, but does not increase the incidence of prostate cancer. Men successfully treated for prostate cancer and diagnosed with hypogonadism are candidates for TRT after a prudent interval for observation, and there is no clinical or laboratory evidence of recurrence of the disease. The risks and benefits of TRT should be clearly understood by the patient and treatment should be carefully monitored. The safety data in this clinical situation are still limited. Maintaining physiological serum levels of testosterone and monitoring PSA in all patients on testosterone replacement is recommended.

\section{WhAT IS THE ROLE OF ART IN THE INCREASED RISK OF CARDIOVASCULAR DISEASE?}

The effects of TRT on cardiovascular risk appear to be associated with the adverse effects on polycythemia, lipid profile and sleep apnea. On the other hand, TRT leads to a decrease in BMI and improves the lipid profile, providing cardiovascular benefit (B). ${ }^{31}$ The maintenance of serum testosterone levels within the normal range does not lead to significant changes in hemoglobin and lipid profiles, decreasing the risk of developing polycythemia and consequently cardiovascular and vascular events $(\mathbf{B}) .^{34,35}$

Hyperinsulinemia and insulin resistance (IR) are essential components of metabolic syndrome, which in turn is associated with an increased cardiovascular risk. TRT improves the components of metabolic syndrome 
in hypogonadal patients with T2DM, reducing the cardiovascular risk $(\mathbf{A}){ }^{36}$

\section{Recommendation}

There is no evidence that TRT per se increases cardiovascular risk. It is recommendable to maintain serum testosterone levels within the normal range so that cardiovascular risk factors such as polycythemia and insulin resistance are minimized, thereby reducing overall cardiovascular risk.

\section{WHAT IS THE RISK OF POLYCYTHEMIA ON ART?}

Secondary polycythemia is a major adverse event of TRT. Several authors have demonstrated its occurrence, and it is related to the maintenance of high serum levels of testosterone, regardless of the treatment time $(\mathbf{B}) .^{31,34,35}$ Therefore, the evidence available so far indicates that the maintenance of serum testosterone levels within the normal average does not lead to polycythemia $(\mathbf{B}){ }^{37}$

\section{Recommendation}

The appearance of polycythemia is directly related to supraphysiological serum testosterone levels. It is recommended to monitor hemoglobin and hematocrit in all patients on TRT and to maintain serum testosterone levels within the normal range to minimize the risk of polycythemia.

\section{WHAT IS THE HEPATOTOXICITY OF ART?}

Hepatotoxicity due to TRT is a rare event limited almost exclusively to the use of oral $17 \alpha$-alkylated preparations such as fluoxymesterone and methyltestosterone, which are highly hepatotoxic and can cause the development of hepatocellular adenomas, liver carcinomas, cholestasis and hemorrhagic cysts of the liver (A). ${ }^{1}$ The long term use of other testosterone preparations does not lead to a change in hepatic function among men with late-onset hypogonadism $(\mathbf{B}){ }^{31,35}$

\section{Recommendation}

$17 \alpha$-alkylated oral preparations such as methyltestosterone and fluoxymesterone present hepatotoxicity. It is not recommended to monitor the liver function of patients on TRT with any other pharmaceutical form.

\section{What IS THE EFFECT OF ART ON SLEeP APNEA? ARE THERE OTHER SIDE EFFECTS?}

Testosterone replacement has been associated with the onset or worsening of sleep apnea in men treated with high doses of testosterone (A). ${ }^{1}$ The administration of testosterone in patients with sleep apnea and erectile dysfunction associated with low testosterone improves sexual symptoms and does not worsen sleep apnea (C). ${ }^{38}$

Gynecomastia is a benign, infrequent and generally reversible complication, a result of the aromatization of testosterone into estradiol in peripheral tissues. Infertility and decreased testicular volume are related to supraphysiological doses of testosterone. Sodium and water retention may occur during replacement and generally present clinical significance in patients with cardiac decompensation, hypertension or renal failure. Skin reactions such as erythema and itching are common with the use of patches. Intramuscular injections may cause local pain, lumps, rashes and boils. Acne, oily skin, increased body hair and skin "flushing" are benign and reversible complications that do not cause major concern (A). ${ }^{1}$

\section{Recommendation}

The side effects of TRT, such as worsening or onset of sleep apnea, gynecomastia, infertility, fluid retention and skin changes are directly related to supraphysiological levels of serum testosterone. It is strongly recommended to maintain serum testosterone levels within the average normal range to minimize the occurrence of these side effects.

\section{What IS THE ROLE OF ART IN METABOLIC SYNDROME?}

The maintenance of serum testosterone levels within the normal range leads to improvements in the markers of metabolic syndrome, such as waist circumference, and increased levels of HDL without causing polycythemia or changes in prostatic parameters. This improvement is not as significant when testosterone levels are maintained at the lower limit of normality (B). ${ }^{37}$ TRT does not depend on the pharmaceutical form of testosterone used or the route of administration and is effective to improve metabolic syndrome parameters; when testosterone levels are maintained within a normal range, however, the improvement is more significant (B). ${ }^{39}$ In T2DM patients, TRT reduces insulin resistance, improves glycemic control, and reduces visceral adiposity and total cholesterol, which are all components of metabolic syndrome (A). ${ }^{36}$

The beneficial effects of ART on metabolic syndrome components appear to be specific to testosterone, given that chronic replacement with DHEA does not improve the secretion or action of insulin and postprandial glycemia in women and elderly men $(\mathbf{B}){ }^{40}$ 


\section{Recommendation}

There is strong evidence that testosterone replacement improves the parameters of metabolic syndrome, especially if serum levels are maintained within normal limits. We recommend maintaining serum testosterone levels within a normal range to aid in the treatment of metabolic syndrome.

\section{HoW SHOULD ART BE ADMINISTERED ORALLY?}

Oral testosterone formulations have been developed to replace injectable forms; however, some disadvantages have been noted, such as variable absorption, low bioavailability due to liver metabolism and the need for 2 to 3 daily doses (D). ${ }^{41}$ Moreover, $17 \alpha$-alkylated derivatives are hepatotoxic $(\mathbf{A})^{1}(\mathbf{D})^{41}$ and have already been withdrawn from the American market (A). ${ }^{19}$ Oral testosterone undecanoate (OTU), the only available oral formulation, is preferably absorbed in chylomicrons, avoiding the primary hepatic passag $(\mathbf{D})^{41}$ and significantly reducing hepatotoxicity (A). ${ }^{17}$ Although most of the studies were flawed due to the small number of participants or the variability of the dose used, the effectiveness of oral ART is questionable. Several authors have shown that the OTU, even at appropriate doses $(160 \mathrm{mg} /$ day $)$ was not effective in improving sexual function, wellbeing, sleep disturbances, cognitive function, mood and quality of life in men with $\operatorname{ADAM}(\mathbf{A}) .^{17,19,20}$ The manufacturer's recommendation is for OTU to be taken during meals, yet there is variability in absorption depending on the composition of the patient's diet (A). ${ }^{19}$

DHEA has been proposed as an alternative oral ART, but the results are controversial. Morales et al. ${ }^{19}$ demonstrated that the ingestion of $50 \mathrm{mg}$ of DHEA orally twice a day, although leading to satisfactory serum levels did not improve the sexual function of men with ADAM (A).${ }^{19}$ On the other hand, replacement with lower doses of DHEA (75 mg/day) improved insulin resistance in the individuals treated $(\mathbf{B}){ }^{42}$

Another way to release testosterone orally is oral mucosa patches which contain $30 \mathrm{mg}$ of testosterone that should be administered twice daily. In general, studies show that this form of ART is capable of maintaining physiological levels of serum testosterone, is safe and well tolerated, and is an interesting option for ART in hypogonadal men $(\mathbf{D})^{41}(\mathbf{B}){ }^{43,44}$

The underreported sublingual form of testosterone administration should be used at a dose of $2.5 \mathrm{mg}$ or $5 \mathrm{mg}, 3$ times a day. It is rapidly absorbed and metabolized, and it does not lead to a sustained increase in serum levels of dihydrotestosterone (DHT) and estradiol(D) ${ }^{41}$

\section{Recommendation}

Oral testosterone undecanoate does not present hepatotoxicity; however, it has proved ineffective in maintaining adequate serum testosterone levels, and has variable absorption between individuals. It is strongly recommended NOT to use oral formulations of testosterone and other androgens as an alternative to TRT.

\section{HoW SHOULD ART BE ADMINISTERED TRANSDERMALLY?}

Transdermal administration of ART includes patches, cutaneous gels and cutaneous solutions.

The patches may be non-scrotal or scrotal, which are thinner and have more effective testosterone absorption than the non-scrotal version. The first presentation of transdermal testosterone was the scrotal patch with release of 4 or $6 \mathrm{mg}$ testosterone/day. It should be applied once daily on depilated scrotal skin $(\mathbf{D}) \cdot{ }^{41}$ The most commonly used patches are non-scrotal, releasing $5 \mathrm{mg}$ /day of testosterone and should be applied once a day on clean and dry glabrous skin $(\mathbf{B}){ }^{43}$

Due to the large number of patches on the international market from different manufacturers, in the opinions of the authors, the tolerability of patients is quite variable due to local adverse effects. They are considered large and uncomfortable to use, and some formulations cause local reactions and exhibit low adhesion that ends up causing low acceptability by patients $(\mathbf{B}) .^{43}$ There are reports of patients who have discontinued treatment due to allergic reactions at the application site, even when using a local corticosteroid ointment $(\mathbf{B}) .^{44}$

On the other hand, the authors are unanimous regarding the efficacy and safety of this type of ART. The transdermal patches available provide physiological and constant levels of serum testosterone, and mimic the circadian rhythm. At a dose of 5 to $7.5 \mathrm{mg} /$ day, changing patches every 48 hours, they are capable of producing a significant improvement in symptoms and quality of life of patients with $\operatorname{ADAM}(\mathbf{A})^{8}(\mathbf{B}) .^{45-47}$ With respect to security, Raynaud et al. ${ }^{44,47}$ reported that the use of transdermal patches showed no negative impact on the hematocrit and lipid profile of patients $(\mathbf{B}),{ }^{45}$ and does not contaminate other people or the environment $(\mathbf{B}){ }^{47}$

Many patients prefer transdermal gels or solutions as they are easy to apply, substantially free of local reactions and do not require injections $(\mathbf{D}) .{ }^{41}$ Testosterone gel formulations available on the health market are available in the concentration of $1 \%$ as "pumps", so that each "puff" releases $1.25 \mathrm{~g}$ of product; individual $2.5 \mathrm{~g}$ and $5 \mathrm{~g}$ packages or single dose tubes containing $5 \mathrm{~g}$ of the product. 
The recommended dose for starting treatment is $5 \mathrm{~g} /$ day, which can be increased up to $10 \mathrm{~g} /$ day $(\mathbf{D}) .^{48}$

Testosterone gel should be applied in the morning on dry skin on the shoulders, arms or abdomen. Patients should wash hands well after application and let the application site dry before putting on clothes. It is recommended to wait 4 hours after application to bathe or swim. The application site should be washed with soap and water if there is direct contact with another person (D). ${ }^{48}$

Testosterone gels at the recommended doses are able to restore the physiological serum testosterone levels but they do not mimic the circadian rhythm $(\mathbf{B})^{46}$ and promote a significant improvement of sexual symptoms and quality of life for patients with $\operatorname{ADAM}(\mathbf{B}) \cdot{ }^{13}$ Even at higher doses $(60 \mathrm{mg})$ applied once daily they promote testosterone levels within the normal range in most patients $(\mathbf{B}) .^{49}$

The association of this type of ART with sildenafil at the maximum dose (100 mg/day) improves erectile dysfunction in hypogonadal men who do not respond to treatment with testosterone alone $(\mathbf{B}){ }_{,}^{50}$ or sildenafil alone $(\mathbf{B}){ }^{49}$

Transdermal testosterone solution at $2 \%$ for axillary use is a new treatment option with characteristics similar to those described for gels $(\mathbf{B}) .^{51}$

A less frequently used form of transdermal ART is the use of dihydrotestosterone gel. Few studies are available for this type of ART. The dose of DHT gel is $70 \mathrm{mg} /$ day for 3 months in patients with ADAM proved to be safe, but with limited effect on physical and cognitive functions. A greater number of long-term studies with a greater numbers of patients are needed to confirm the safety and efficacy of DHT as a treatment option for ART (A). ${ }^{52}$

\section{Recommendation}

There is strong evidence that transdermal testosterone replacement (patches, gels or solution) is safe and effective in addition to being the most physiological. Patches, in turn, are capable of mimicking the circadian rhythm of testosterone secretion; however, these pharmaceutical forms are not available in our country. The use of transdermal TRT is recommended for being the most physiological.

\section{IS THERE A DIFFERENCE BETWEEN THE COMMERCIAL GEL AND COMPOUND GEL?}

There are no scientific studies comparing commercial gels and compound ones. Only two studies have compared gels that are produced in two different countries that are not widely available in the global market. The ointment produced in Japan was used on 50 patients with ADAM at a dose of $3 \mathrm{mg}$ twice daily on the skin of the scrotum for 12 weeks and caused a physiological increase in total and free testosterone with no severe adverse effects $(\mathbf{B}) .^{53}$ The gel produced in Germany was applied both on scrotal and non-scrotal skin and removed after 10 minutes in hypogonadal men. There was better tolerability than the commercial gels, less chance of interpersonal transfer due to early removal of the gel and an effective increase in testosterone levels $(\mathbf{B}) .^{54}$

\section{Recommendation}

Studies with a good level of evidence using noncommercial testosterone gel are still scarce. The use of a testosterone gel preparation without proven efficacy and safety is not recommended.

\section{How SHOULd ART BE ADMINISTERED USING SUBCUTANEOUS IMPLANTS?}

Subcutaneous implants are composed of 1,200 mg of crystallized testosterone, are generally more accepted by patients, but require a surgical procedure and if not performed by experienced physicians may have high rates of extrusion $(\mathbf{B}) .^{43}$ The implants are changed every 3-6 months and are considered safe in long-term studies(B). ${ }^{34}$ Experience with this type of ART is still limited in the literature.

\section{Recommendation}

Studies with a good level of evidence for this form of TRT are scarce and this pharmaceutical form is not available in our country. We recommend not using subcutaneous testosterone implants until a larger number of studies on safety and efficacy can be found, as the extrusion rate appears to be high.

\section{How SHOULd INJeCtable ART be ADMINISTERED?}

Injectable testosterone preparations have been widely used for many years. They consist of various testosterone esters, combined or otherwise, namely, propionate, phenylpropionate, enanthate, cypionate, decanoate, isocaproate and undecanoate $(\mathbf{D}) .{ }^{41}$ All formulations are available in Brazil, except those containing testosterone enanthate $(\mathbf{B}){ }^{55}$ All injections with testosterone esters have short action, except for testosterone undecanoate depot (TUD).

The short acting formulations must be injected every 14-21 days while the long acting ones should be applied every 6 weeks at the start of the treatment and every 12 weeks thereafter $(\mathbf{D})^{41}(\mathbf{B}) .{ }^{55}$ Replacement therapy using short acting testosterone for a short period of time has proven effective and safe, improving sexual function in patients without increasing hematocrit or PSA $(\mathbf{D})^{41}(\mathbf{B}){ }^{55,56}$ 
In relation to TUD, in order to achieve adequate serum levels, the second injection should be given after 6 to 10 weeks, with the interval subsequently extended up to $10-14$ weeks (B). ${ }^{57}$ It is recommended to apply the second injection after 6 weeks and then one injection every 12 weeks $(\mathbf{B})^{15,58,59}(\mathbf{C}){ }^{16}$ It is usually well tolerated and changes in the actions and side effects are more frequently observed in obese patients (B). ${ }^{57}$

There are two presentations of TUD - the formulation available in the USA contains $750 \mathrm{mg}$ of TUD, maintains serum testosterone levels within the normal range during an interval of 10 weeks between doses and has proven to be safe and effective (B). ${ }^{60} 1000 \mathrm{mg}$ TUD is available in Europe and South America, including Brazil. Its pharmacokinetics enables one injection every 12 weeks to be able to maintain physiological levels of serum testosterone without causing peaks above normal (B).$^{55}$

\section{Recommendation}

Although not the most physiological, the short acting injectable forms have proven safe and effective over time, but with the disadvantage of fortnightly application. Besides providing physiological levels of testosterone, the pharmacokinetics of TUD enables quarterly applications, but with the disadvantage of having a high cost. Among the available injectable forms, the use of TUD to maintain physiological levels of testosterone is recommended in order to minimize side effects with fewer injections.

\section{Are there comparative studies between THEM? WHAT IS THE BEST FORM OF ART?}

The criterion to assess efficacy of ART is that testosterone levels are maintained within normal limits during the 24 hour interval between two applications (WHO, 1992). The various testosterone formulations differ mainly in relation to the route of administration and the pharmacokinetics of the components, causing variability in their effectiveness.

Several authors have carried out comparative studies between them. In men with ADAM and metabolic syndrome, treatment with TUD demonstrated greater effect on sexual symptoms and metabolic syndrome parameters than testosterone gel $(\mathbf{A})^{36}(\mathbf{B}){ }^{38}$ while the safety parameters were similar in both modalities $(\mathbf{B}) .^{38}$

A 30 weeks follow-up of patients who used short acting TUD or testosterone enanthate showed that the effects of the two formulations were similar, although treatment with TUD requires only four injections per year to maintain testosterone levels within the physiological range. Polycythemia was not observed in patients who used $\operatorname{TUD}(\mathbf{B}){ }^{15}$
The comparison of the pharmacokinetics and tolerability of oral patches and non-scrotal skin patches showed that the oral system promotes physiologic levels of testosterone at a higher percentage of the day than conventional skin patch systems $(\mathbf{B}) .{ }^{43}$

A comparative study between the ART and skin patches at the maximum dose ( $4.8 \mathrm{mg}$ every 48 hours -2 patches) and short acting testosterone ( $250 \mathrm{mg}$ every 3 weeks) showed that the percentage of patients who maintained stable and physiological levels of testosterone was higher in those using patches than in patients who used injectable testosterone, with high variability in the testosterone levels $(\mathbf{B}){ }^{44}$

The effects of the three injectable formulations available in Brazil (two with short acting esters and one with TUD) were compared and it was found that the three formulations are effective in raising testosterone levels and clinically improving hypogonadal patients, with TUD even though, more expensive - being clinically and laboratorially more effective. The three options were shown to be safe without significantly increasing hematocrit, hemoglobin and PSA $(\mathbf{B}) .^{55}$

The treatment of hypogonadal patients with short acting injectable testosterone at doses of $100-200 \mathrm{mg} /$ week who were subsequently submitted to treatment with testosterone gel at a dose of $5-10 \mathrm{~g}$ gel/day demonstrated that testosterone gel produced stable concentrations of testosterone and improved the quality of life of patients compared to injectable testosterone $(\mathbf{B}) .{ }^{61}$

The usual dose of testosterone gel determined physiological levels of serum testosterone at physiological levels that were not significantly changed after association of any dose of oral testosterone $(\mathbf{B}){ }^{62}$

Therefore, the comparative analysis between the main types of ART has shown that all are safe, and transdermal forms and TUD are the most physiological.

\section{Recommendation}

The comparative analysis between the main types of ART has shown that all are safe, and transdermal forms and TUD are the most physiological. The use of TUD or testosterone gel is recommended, whenever possible, for ART in hypogonadal patients.

\section{WHAT IS THE ROLE OF PROLONGED ACTION TESTOSTERONE?}

There are two formulations of long acting testosterone undecanoate, one presented in $750 \mathrm{mg}$ vials, available in the US, and another with $1000 \mathrm{mg}$ vials, available in Europe and South America. Both are well tolerated by patients, are able 
to maintain physiological and stable levels of serum testosterone and DHT $(\mathbf{B})^{59}$ and have similar effectiveness and safety as other injectable forms $(\mathbf{B})^{58,63}(\mathbf{D}) \cdot{ }^{64}$ The advantage of TUD is that with only 4-5 injections per year testosterone levels can be maintained at physiological levels $(\mathbf{B}) .^{15}$

TUD appears to have a role in the treatment of metabolic syndrome. A follow-up of patients with metabolic syndrome and ADAM using TUD for a period of one year showed that testosterone levels were restored to the normal average value. There was a significant improvement in sexual symptoms, metabolic syndrome parameters and body composition. There was no change in PSA, blood glucose and liver function, and the levels of hemoglobin and hematocrit did not exceed the upper limit of a normal range $(\mathbf{A})^{36}(\mathbf{B})^{38}(\mathbf{C}){ }^{16}$

The increase in blood pressure, hematocrit $>50 \%$ and worsening lipid profile that may occur in a small number of patients were related to the number of CAG repeats of the androgen receptor and the presence of obesity $(\mathbf{B}){ }^{57}$ ART with long acting TU proved to be safe for a period of 24 months of treatment.

\section{Recommendation}

The main role of long acting testosterone is maintenance of physiological serum testosterone levels with a lower number of applications and consequently lower rate of side effects.

\section{IS THERE A DIFFERENCE IN ABSORPTION BETWEEN THE MANY PHARMACOLOGICAL PREPARATIONS?}

Each formulation has a distinct characteristic, depending on the presentation, dose and pharmacokinetics. The testosterone esters used in short acting injectable formulations are derived from fatty acids and depend on the esters being releases from the oily vehicle and the hydrolysis of these esters in order to release of testosterone into the circulation. The pharmacokinetics are determined in part by the size of the side chain, hence the wide variation in the use of different esters modulates the level of circulating testosterone $(\mathbf{D}){ }^{41}$

The testosterone levels obtained with short acting injectable forms are unstable (B), ${ }^{55,61}$ whereas long acting injectable forms provide more stable levels.

In the oral formulations, absorption is variable and bioavailability is generally poor due to the effect of first passing through the liver. Oral testosterone undecanoate is preferentially absorbed by chylomicrons, avoiding the liver. However, the testosterone level is sub-optimal and it must be taken various times per day $(\mathbf{D}) .{ }^{41}$
The oral mucosa patches and sublingual formulation are not often used. The first seems to have a good absorption similar to that of gels, but a shorter half-life, requiring use twice per day $(\mathbf{B}) .{ }^{43}$ Transdermal patches are presented in formulations that vary in size and thus the dose of testosterone. Studies indicate that absorption is efficient and reaches equilibrium in 48 hours (B).$^{44}$ The absorption of scrotal patches is more efficient because the skin is thinner and increased production of DHT occurs because the amount of 5 alpha reductase in that region is higher $(\mathbf{D}){ }^{41}$ The evening application of the patch produces serum testosterone mimicking the circadian rhythm of healthy men, while the application of the gel in the morning produces physiological and stable serum testosterone levels (B) ${ }^{15,46,55,61}$ which shows the different absorption mechanisms between the two formulations.

\section{Recommendation}

The differences in absorption between the various pharmaceutical forms depend on the presentation, dose and pharmacokinetics. Testosterone levels obtained by short acting injectable forms and oral forms are more unstable and less physiological. On the other hand, the levels obtained by means of injection and for long acting transdermal forms are stable and physiological. The only pharmaceutical form that mimics the circadian rhythm is that of scrotal patch. The preferential use of the long acting injectable form or transdermal forms is recommended for ART.

\section{WHAT ARE THE CONTRAINDICATIONS FOR ART?}

An increased risk of cardiovascular events has been observed in elderly patients with ADAM associated with other chronic diseases.

The administration of testosterone is contraindicated in men with prostate or breast cancer, in men with palpable prostate nodules or PSA greater than $4 \mathrm{ng} / \mathrm{mL}$ or $3 \mathrm{ng} / \mathrm{mL}$ in high risk patients $(\mathbf{D}){ }^{41,48}$

ART is recommended to be applied with caution in men with benign prostate hypertrophy and mild or moderate urinary symptoms, while men with severe urinary symptoms should undergo urologic evaluation before starting treatment.

Testosterone should not be used in men with hematocrit $>50 \%$ or patients with grade III or IV CHF. Men with moderate or severe obstructive sleep apnea should be evaluated by a specialist before starting ART $(\mathbf{D}){ }^{48}$ 


\section{Recommendation}

There are few studies with high levels of evidence in relation to the contraindications of testosterone replacement. It is weakly recommendable to not use testosterone in patients with prostate or breast cancer with PSA $>4 \mathrm{ng} / \mathrm{mL}$ or $3 \mathrm{ng} / \mathrm{mL}$ in high risk patients or those with palpable prostate nodule, hematocrit greater than $50 \%$ or in patients with grade II or IV CHF.

\section{References}

1. Martits AM, Costa EMF. Late onset male hypogonadism or andropause. Rev Assoc Med Bras 2004;50:358-9.

2. Wang C, Nieschlag E, Swerdloff RS, Behre H, Hellstrom WJ, Gooren LJ, et al. ISA, ISSAM, EAU, EAA and ASA recommendations: investigation, treatment and monitoring of late-onset hypogonadism in males. Aging Male 2009;12:05 AM-12.

3. Merza Z, Blumsohn A, Mah PM, Meads DM, McKenna SP, Wylie K, et al. Double-blind placebo-controlled study of testosterone patch therapy on bone turnover in men with borderline hypogonadism. Int J Androl 2006;29:381-91.

4. Srinivas-Shankar U, Roberts SA, Connolly MJ, O’Connell MD, Adams JE, Oldham JA, et al. Effects of testosterone on muscle strength, physical function, body composition, and quality of life in intermediate-frail and frail elderly men: a randomized, double-blind, placebo-controlled study. J Clin Endocrinol Metab 2010;95:639-50.

5. Sullivan DH, Roberson PK, Johnson LE, Bishara O, Evans WJ, Smith ES, et al. Effects of muscle strength training and testosterone in frail elderly males. Med Sci Sports Exerc 2005;37:1664-72.

6. Allan CA, Strauss BJ, Burger HG, Forbes EA, McLachlan RI. Testosterone therapy prevents gain in visceral adipose tissue and loss of skeletal muscle in nonobese aging men. J Clin Endocrinol Metab 2008;93:139-46.

7. Kapoor D, Goodwin E, Channer KS, Jones TH. Testosterone replacement therapy improves insulin resistance, glycaemic control, visceral adiposity and hypercholesterolaemia in hypogonadal men with type 2 diabetes. Eur J Endocrinol 2006;154:899-906.

8. Katznelson L, Robinson MW, Coyle CL, Lee H, Farrell CE. Effects of modest testosterone supplementation and exercise for 12 weeks on body composition and quality of life in elderly men. Eur J Endocrinol 2006;155:867-75.

9. Kalinchenko SY, Tishova YA, Mskhalaya GJ, Gooren LJ, Giltay EJ, Saad F. Effects of testosterone supplementation on markers of the metabolic syndrome and inflammation in hypogonadal men with the metabolic syndrome: the double-blinded placebo-controlled Moscow study. ClinEndocrinol (Oxf). 2010;73:602-12.

10. Giannoulis MG, Sonksen PH, Umpleby M, Breen L, Pentecost C, Whyte M, et al. The effects of growth hormone and/or testosterone in healthy elderly men: a randomized controlled trial. J Clin Endocrinol Metab 2006;91:477-84.

11. Sattler FR, Castaneda-Sceppa C, Binder EF, Schroeder ET, Wang Y, Bhasin $\mathrm{S}$, et al. Testosterone and growth hormone improve body composition and muscle performance in older men. J Clin Endocrinol Metab 2009;94:19912001.

12. Isidori AM, Giannetta E, Gianfrilli D, Greco EA, Bonifacio V, Aversa A, et al. Effects of testosterone on sexual function in men: results of a metaanalysis. ClinEndocrinol (Oxf) 2005;63:381-94.

13. Chiang HS, Hwang TI, Hsui YS, Lin YC, Chen HE, Chen GC, et al. Transdermal testosterone gel increases serum testosterone levels in hypogonadal men in Taiwan with improvements in sexual function. Int J Impot Res 2007;19:411-7.

14. Allan CA, Forbes EA, Strauss BJ, McLachlan RI. Testosterone therapy increases sexual desire in ageing men with low-normal testosterone levels and symptoms of androgen deficiency. Int J Impot Res 2008;20:396-401.

15. Jockenhövel F, Minnemann T, Schubert M, Freude S, Hübler D, Schumann $\mathrm{C}$, et al. Comparison of long-acting testosterone undecanoate formulation versus testosterone enanthate on sexual function and mood in hypogonadal men. Eur J Endocrinol 2009;160:815-9.

16. Saad F, Gooren L, Haider A, Yassin A. An exploratory study of the effects of 12 month administration of the novel long-acting testosterone undecanoate on measures of sexual function and the metabolic syndrome. Arch Androl 2007;53:353-7.

17. Legros JJ, Meuleman EJ, Elbers JM, Geurts TB, Kaspers MJ, Bouloux PM, et al. Oral testosterone replacement in symptomatic late-onset hypogonadism: effects on rating scales and general safety in a randomized, placebo-controlled study. Eur J Endocrinol 2009;160:821-31.

18. Shamloul R, Ghanem H, Fahmy I, El-Meleigy A, Ashoor S, Elnashaar A, et al. Testosterone therapy can enhance erectile function response to sildenafil in patients with PADAM: a pilot study. J Sex Med 2005;2:559-64.

19. Morales A, Black A, Emerson L, Barkin J, Kuzmarov I, Day A. Androgens and sexual function: a placebo-controlled, randomized, double-blind study of testosterone vs. dehydroepiandrosterone in men with sexual dysfunction and androgen deficiency. Aging Male 2009;12:104-12.

20. Haren MT, Wittert GA, Chapman IM, Coates P, Morley JE. Effect of oral testosterone undecanoate on visuospatial cognition, mood and quality of life in elderly men with low-normal gonadal status. Maturitas 2005;50:124-33.

21. Vaughan C, Goldstein FC, Tenover JL. Exogenous testosterone alone or with finasteride does not improve measurements of cognition in healthy older men with low serum testosterone. J Androl 2007;28:875-82.

22. Young LA, Neiss MB, Samuels MH, Roselli CE, Janowsky JS. Cognition is not modified by large but temporary changes in sex hormones in men. J Clin Endocrinol Metab 2010;95:280-8.

23. Corona G, Monami M, Rastrelli G, Aversa A, Sforza A, Lenzi A, et al. Type 2 diabetes mellitus and testosterone: a meta-analysis study. Int J Androl 2011;34( Pt1):528-40.

24. Herzog AG, Farina EL, Drislane FW, Schomer DL, Smithson SD, Fowler $\mathrm{KM}$, et al. A comparison of anastrozole and testosterone versus placebo and testosterone for treatment of sexual dysfunction in men with epilepsy and hypogonadism. Epilepsy Behav 2010;17:264-71.

25. Basu R, Dalla Man C, Campioni M, Basu A, Nair KS, Jensen MD, et al. Two years of treatment with dehydroepiandrosterone does not improve insulin secretion, insulin action, or postprandial glucose turnover in elderly men or women. Diabetes 2007;56:753-66.

26. Rhoden EL, Morgentaler A. Influence of demographic factors and biochemical characteristics on the prostate-specific antigen (PSA) response to testosterone replacement therapy. Int J Impot Res 2006;18:201-5.

27. Sarosdy MF. Testosterone replacement for hypogonadism after treatment of early prostate cancer with brachytherapy. Cancer 2007;109:536-41.

28. Morales A, Black AM, Emerson LE. Testosterone administration to men with testosterone deficiency syndrome after external beam radiotherapy for localized prostate cancer: preliminary observations. BJU Int 2009;103:62-4

29. Khera M, Grober ED, Najari B, Colen JS, Mohamed O, Lamb DJ, et al. Testosterone replacement therapy following radical prostatectomy. J Sex Med 2009;6:1165-70.

30. Leibowitz RL, Dorff TB, Tucker S, Symanowski J, Vogelzang NJ. Testosterone replacement in prostate cancer survivors with hypogonadal symptoms. BJU Int 2010;105:1397-401.

31. Coward RM, Simhan J, Carson CC $3^{\text {rd }}$. Prostate-specific antigen changes and prostate cancer in hypogonadal men treated with testosterone replacement therapy. BJU Int 2009;103:1179-83.

32. Marks LS, Mazer NA, Mostaghel E, Hess DL, Dorey FJ, Epstein JI, et al. Effect of testosterone replacement therapy on prostate tissue in men with late-onset hypogonadism: a randomized controlled trial. JAMA 2006;296:2351-61.

33. Daniels NA, Nielson CM, Hoffman AR, Bauer DC; Osteoporotic Fractures In Men (MrOS) Study Group. Sex hormones and the risk of incident prostate cancer. Urology 2010;76:1034-40.

34. Ip FF, di Pierro I, Brown R, Cunningham I, Handelsman DJ, Liu PY. Trough serum testosterone predicts the development of polycythemia in hypogonadal men treated for up to 21 years with subcutaneous testosterone pellets. Eur J Endocrinol 2010;162:385-90.

35. Yamaguchi K, Ishikawa T, Chiba K, Fujisawa M. Assessment of possible effects for testosterone replacement therapy in men with symptomatic lateonset hypogonadism. Andrologia 2011;43:52-6.

36. Kapoor D, Goodwin E, Channer KS, Jones TH. Testosterone replacement therapy improves insulin resistance, glycaemic control, visceral adiposity and hypercholesterolaemia in hypogonadal men with type 2 diabetes. Eur J Endocrinol 2006;154:899-906.

37. Saad F, Gooren L, Haider A, Yassin A. Effects of testosterone gel followed by parenteral testosterone undecanoate on sexual dysfunction and on features of the metabolic syndrome. Andrologia 2008;40:44-8.

38. Zhuravlev VN, Frank MA, Gomzhin AI. Sexual functions of men with obstructive sleep apnoea syndrome and hypogonadism may improve upon testosterone administration: a pilot study. Andrologia 2009;41:193-5. 
39. Saad F, Gooren LJ, Haider A, Yassin A. A dose-response study of testosterone on sexual dysfunction and features of the metabolic syndrome using testosterone gel and parenteral testosterone undecanoate. J Androl 2008;29: 102-5.

40. Basu R, Dalla Man C, Campioni M, Basu A, Nair KS, Jensen MD, et al. Two years of treatment with dehydroepiandrosterone does not improve insulin secretion, insulin action, or postprandial glucose turnover in elderly men or women. Diabetes 2007;56:753-66

41. Ebert T, Jockenhövel F, Morales A, Shabsigh R. The current status of therapy for symptomatic late-onset hypogonadism with transdermal testosterone gel.EurUrol 2005;47:137-46.

42. Yamada Y, Sekihara H, Omura M, Yanase T, Takayanagi R, Mune T, et al. Changes in serum sex hormone profiles after short-term low-dose administration of dehydroepiandrosterone (DHEA) to young and elderly persons. Endocr J 2007;54:153-62.

43. Korbonits M, Slawik M, Cullen D, Ross RJ, Stalla G, Schneider H, et al. A comparison of a novel testosterone bioadhesivebuccal system, striant, with a testosterone adhesive patch in hypogonadal males. J Clin Endocrinol Metab 2004;89:2039-43

44. Raynaud JP, Legros JJ, Rollet J, Augès M, Bunouf P, Sournac M, et al. Efficacy and safety of a new testosterone-in-adhesive matrix patch applied every 2 days for 1 year to hypogonadal men. J Steroid Biochem Mol Biol 2008;109:168-76.

45. Daniell HW, Lentz R, Mazer NA. Open-label pilot study of testosterone patch therapy in men with opioid-induced androgen deficiency. J Pain 2006;7:200-10.

46. Mazer N, Bell D, Wu J, Fischer J, Cosgrove M, Eilers B, et al. Comparison of the steady-state pharmacokinetics, metabolism, and variability of a transdermal testosterone patch versus a transdermal testosterone gel in hypogonadal men. J Sex Med 2005;2:213-26.

47. Raynaud JP, Aumonier C, Gualano V, Betea D, Beckers A. Pharmacokinetic study of a new testosterone-in-adhesive matrix patch applied every 2 days to hypogonadal men. J Steroid Biochem Mol Biol 2008;109:177-84.

48. Lakshman KM, Basaria S. Safety and efficacy of testosterone gel in the treatment of male hypogonadism. ClinInterv Aging 2009;4:397-412.

49. Meikle AW, Matthias D, Hoffman AR. Transdermal testosterone gel: pharmacokinetics, efficacy of dosing and application site in hypogonadal men. BJU Int 2004;93:789-95.

50. Greenstein A, Mabjeesh NJ, Sofer M, Kaver I, Matzkin H, Chen J. Does sildenafil combined with testosterone gel improve erectile dysfunction in hypogonadal men in whom testosterone supplement therapy alone failed? J Urol 2005; 173:530-2.

51. Wang C, Ilani N, Arvert S, McLachlan RI, Soulis T, Watkinson A. Efficacy and safety of the $2 \%$ formulation of testosterone topical solution applied to the axillae in androgen-deficient men. Clin Endocrinol (Oxf). 2011;75:836-43.

52. Ly LP, Jimenez M, Zhuang TN, Celermajer DS, Conway AJ, Handelsman DJ. A double-blind, placebo-controlled, randomized clinical trial of transdermal dihydrotestosterone gel on muscular strength, mobility, and quality of life in older men with partial androgen deficiency. J Clin Endocrinol Metab 2001;86: 4078-88.

53. Amano T, Imao T, Takemae K, Iwamoto T, Yamakawa K, Baba K, et al. Profile of serum testosterone levels after application of testosterone ointment (glowmin) and its clinical efficacy in late-onset hypogonadism patients. J Sex Med 2008;5:1727-36.

54. Kühnert B, Byrne M, Simoni M, Köpcke W, Gerss J, Lemmnitz G, et al. Testosterone substitution with a new transdermal, hydroalcoholic gel applied to scrotal or non-scrotal skin: a multicentre trial. Eur J Endocrinol 2005; 153:317-26.

55. Hohl A, Marques MO, Coral MH, Walz R. Evaluation of late-onset hypogonadism (andropause) treatment using three different formulations of injectable testosterone. Arq Bras Endocrinol Metabol 2009;53:989-95.

56. Andrade ES Jr, Clapauch R, Buksman S. Short term testosterone replacement therapy improves libido and body composition. Arq Bras Endocrinol Metabol 2009;53:996-1004.

57. Zitzmann M, Nieschlag E. Androgen receptor gene CAG repeat length and body mass index modulate the safety of long-term intramuscular testosterone undecanoate therapy in hypogonadal men. J Clin Endocrinol Metab 2007;92: 3844-53.

58. Haider A, Gooren LJ, Padungtod P, Saad F. A safety study of administration of parenteral testosterone undecanoate to elderly men over minimally 24 months. Andrologia 2010;42:349-55.

59. Yassin AA, Saad F. Plasma levels of dihydrotestosterone remain in the normal range in men treated with long-acting parenteral testosterone undecanoate. Andrologia 2007;39:181-4.

60. Morgentaler A, Dobs AS, Kaufman JM, Miner MM, Shabsigh R, Swerdloff $\mathrm{RS}$, et al. Long acting testosterone undecanoate therapy in men with hypogonadism: results of a pharmacokinetic clinical study. J Urol 2008;180: 2307-13.

61. Scott JD, Wolfe PR, Anderson P, Cohan GR, Scarsella A. Prospective study of topical testosterone gel (AndroGel) versus intramuscular testosterone in testosterone-deficient HIV-infected men. HIV Clin Trials 2007;8:412-20.

62. Birzniece V, Meinhardt UJ, Handelsman DJ, Ho KK. Testosterone stimulates extra-hepatic but not hepatic fat oxidation (Fox): comparison of oral and transdermal testosterone administration in hypopituitary men. Clin Endocrinol (Oxf) 2009;71:715-21.

63. Wang C, Harnett M, Dobs AS, Swerdloff RS. Pharmacokinetics and safety of long-acting testosterone undecanoate injections in hypogonadal men: an 84-week phase III clinical trial. J Androl 2010;31:457-65.

64. Agledahl I, Brodin E, Svartberg J, Hansen JB. Impact of long-term testosterone treatment on plasma levels of free TFPI and TF-induced thrombin generation ex vivo in elderly men with low testosterone levels. Thromb Haemost 2009;102: 945-50. 